\title{
22 Epigenetics, Nutrition, and Infant Health
}

\author{
Philip T. James, Matt J. Silver, \\ and Andrew M. Prentice
}

\section{CONTENTS}

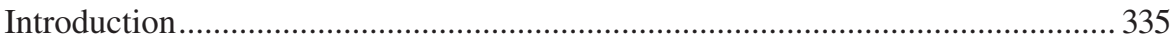

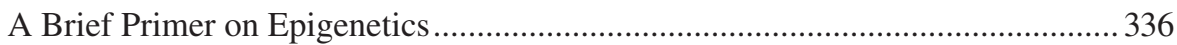

Time Points of Plasticity in the Epigenome ....................................................... 337

Which Parts of the Epigenome Are Most Susceptible to Environmental

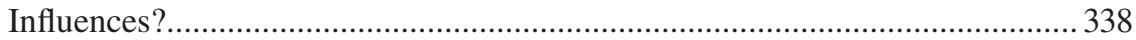

Epigenetics and the Developmental Origins of Health and Disease........................339

Maternal Nutrition Exposures and the Infant Epigenome .....................................340

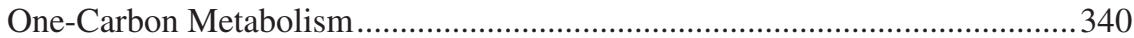

Studies Investigating One-Carbon Metabolites and Epigenetics ...................... 343

Polyunsaturated Fatty Acids.......................................................................... 345

Body Mass Index....................................................................................... 346

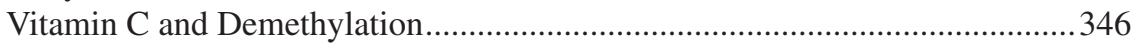

Phenotypes Associated with the Impact of Maternal Exposures on the Infant

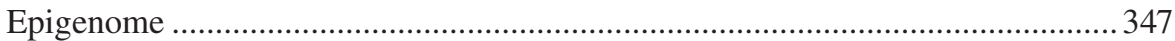

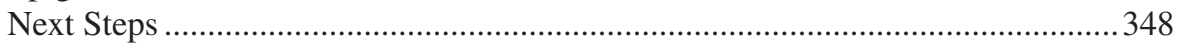

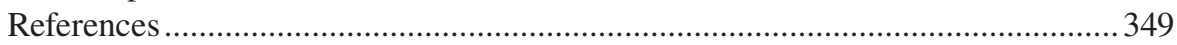

\section{INTRODUCTION}

The field of epigenetics is currently garnering a great deal of interest, exploring how our very molecular makeup in the form of modifications to the genome can be altered by factors as diverse as aging, disease, nutrition, stress, alcohol, and exposure to pollutants. Epigenetic changes have previously been implicated in the etiology of a variety of diseases [1], notably in the development of certain cancers [2], and inherited growth disorder syndromes [3], but the exploration of epigenetics' role in fetal programming is still in its infancy. This chapter focuses on how nutritional exposures during pregnancy may affect the infant epigenome, and the impact that such modifications may have on the long-term health of the child. We start by describing some keys concepts in epigenetics and discuss windows of epigenetic plasticity in the context of the developmental origins of health and disease (DOHaD) hypothesis. We then review some of the key mechanisms by which nutrition can affect the epigenome, with a particular focus on the role of one-carbon metabolism. We finish by outlining some of the child health outcomes that have been linked to epigenetic 
dysregulation, and discuss possible next steps that need to be realized if insights into the basic science of epigenetics are to be translated into tangible public health benefits.

\section{A BRIEF PRIMER ON EPIGENETICS}

Epigenetic processes describe changes to the genome that can alter gene expression without changing the underlying DNA sequence [4]. These changes are mitotically heritable, and involve the interplay of DNA methylation, histone modifications, and RNA-based mechanisms (Figure 22.1). DNA methylation most commonly occurs at loci where a cytosine is found next to a guanine on a DNA strand along its linear sequence, hence termed cytosine-phosphate-guanine or $\mathrm{Cp} G$ sites. It involves the covalent bonding of a methyl $\left(\mathrm{CH}_{3}\right)$ group to the cytosine at the $5^{\prime}$ carbon position to form 5-methylcytosine. CpGs found in high densities are termed $C p G$ islands. Roughly two-thirds of human genes contain $\mathrm{CpG}$ islands in their promoter regions, although repetitive elements in the genome can also contain many CpG sites [5]. CpGs are generally methylated in nonpromoter regions and unmethylated at promoter regions. Methylation at $\mathrm{CpG}$ sites in promoters is usually associated with transcriptional silencing, although not consistently [6]. Although methylation is the most studied chemical alteration to date, others modifications (e.g., hydroxymethylation) can occur at cytosine bases [7].

DNA methylation is catalyzed by DNA methyltransferases (DNMTs). Mammals have three types of DNMT. DNMT1 recognizes hemimethylated DNA; therefore, after DNA replication and cell division, it methylates the newly synthesized strand to

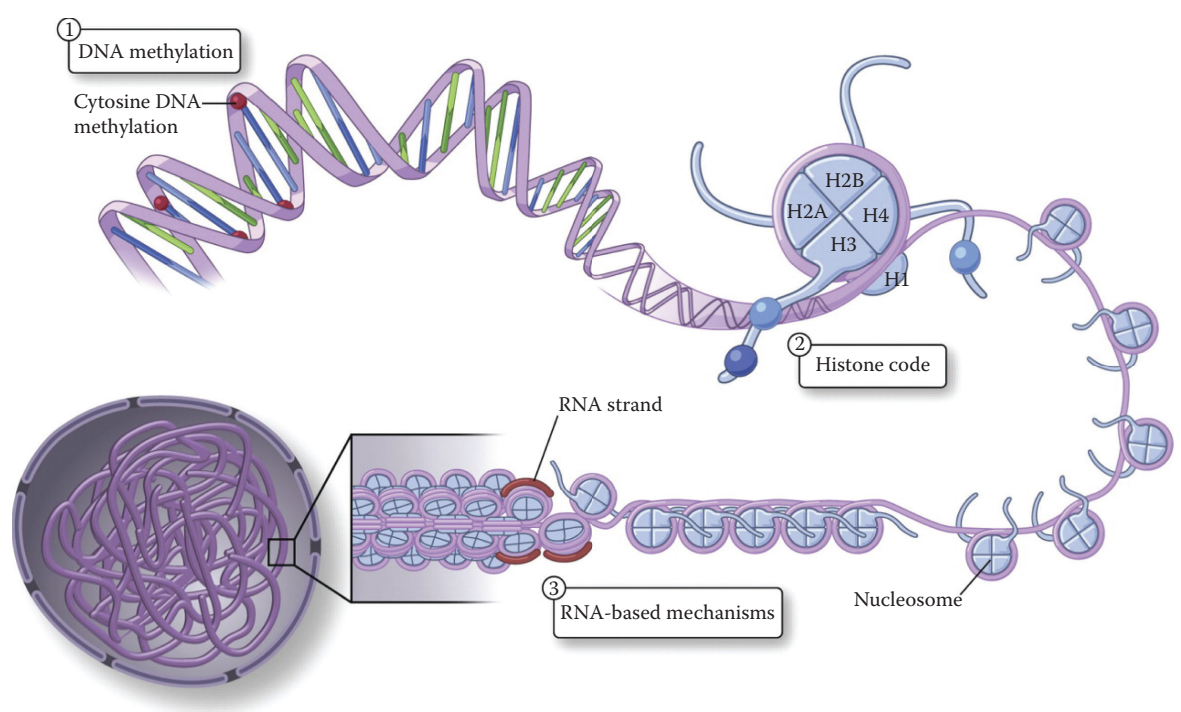

FIGURE 22.1 An overview of epigenetic mechanisms. (From Yan MS-C, Matouk CC, Marsden PA, J Appl Physiol 2010, 109:916-26.) 
maintain methylation patterns of the original template strand (maintenance methylation). DNMT3a and 3b appear to primarily methylate fully unmethylated $\mathrm{CpG}$ sites (de novo methylation) [7]. DNA methylation is important for a host of biological processes, including transcriptional silencing, $\mathrm{X}$-chromosome inactivation, genomic imprinting, and the maintenance of cellular identity by enabling tissue-specific gene expression [8].

DNA is tightly woven around histone proteins, forming compact complexes of DNA and protein called nucleosomes. Nucleosomes, in turn, are packed together to form chromatin. Within the nucleosomes, the histone proteins are arranged in an eight-part formation, comprising two copies each of histones $\mathrm{H} 2 \mathrm{~A}, \mathrm{H} 2 \mathrm{~B}, \mathrm{H} 3$, and H4. Histone modifications involve various posttranslational chemical alterations to the amino acids of the histone tails, including acetylation of lysine, methylation of lysine and arginine, phosphorylation of serine and threonine, and the ubiquitination of lysine [9]. There are several mechanisms by which chemical modifications of $\mathrm{CpG}$ sites and histones are thought to influence gene expression. The methyl group from 5-methylcytosine may block transcription factors either directly or through the recruitment of a methyl-binding protein. Alternatively, the DNMT enzymes acting on $\mathrm{CpG}$ sites may be physically linked to other enzymes, which bring about histone methylation and deacetylation [10]. Although chromatin remodeling is intricately controlled, a simplified summary is that the hyperacetylation of histones and hypomethylation of histones and CpGs is associated with a euchromatin (open) configuration, generally associated with facilitation of transcriptional activity. Conversely, hypoacetylation of histones and hypermethylation of histones and CpGs is associated with a heterochromatin (closed) structure and transcriptional repression [11].

Although noncoding RNAs do not code for proteins, many are functional and may affect gene expression [12]. Of those that influence gene expression, microRNAs (miRNAs) have been the most studied to date. These are short pieces ( 22 nucleotides) of RNA that affect the epigenome through binding to target mRNAs controlling the expression of key regulators such as DMNTs and histone deacetylases [13]. In turn, $\mathrm{CpG}$ methylation and histone modifications can influence the transcription of certain miRNA classes [14]. MicroRNAs may also affect gene expression directly by binding to messenger RNAs, repressing their translation [14].

\section{TIME POINTS OF PLASTICITY IN THE EPIGENOME}

Times of increased cell turnover, such as during fetal development and infancy, may be particularly susceptible both to epigenetic errors and to environmental influences [15]. In this chapter, we focus on DNA methylation and the in utero period, to include periconception, since this falls within the first 1,000 days window, and is also a period of exceptionally rapid cell differentiation and complex epigenetic remodeling (Figure 22.2). In the first 48 hours after fertilization, there is rapid (active) demethylation of the paternal genome and a slower (passive) demethylation of the maternal genome [16]. Erasing the epigenetic marks in the zygote prior to the blastocyst stage is important to enable pluripotency of the developing cells [8]. Imprinted genes and some retrotransposons (defined later) are known to resist demethylation at this stage [17]. Remethylation then occurs in tissue-specific patterns after implantation, 


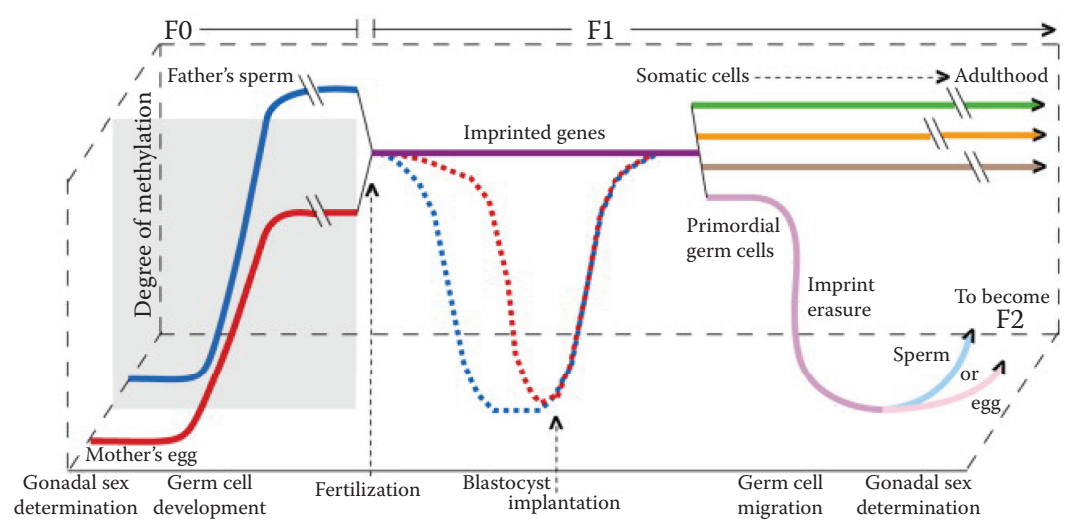

FIGURE 22.2 Epigenetic remodeling in embryogenesis. (From Perera F, Herbstman J, Reprod Toxicol 2011, 31:363-73.)

during the process of gastrulation and differentiation of the somatic cells throughout pregnancy. A second wave of demethylation occurs during the epigenetic reprogramming of primordial germ cells (PGCs) in the developing embryo at the point of their migration to the genital ridge [18]. Parental imprints are erased at this stage in preparation for the laying down of sex-specific imprints in the PGCs. Remethylation of sperm cells occurs before the birth of the child, and in oocytes over the duration of their maturation [18]. The periconceptional period is therefore one of huge dynamism in the methylome, representing a window in which epigenetic errors could have significant consequences for the health of the child.

\section{Which Parts of the Epigenome Are Most Susceptible TO ENVIRONMENTAL INFLUENCES?}

There are some regions of the genome that demonstrate increased interindividual epigenetic variation, and may be particularly vulnerable to the impact of environmental influences [19]. These include imprinted genes, metastable epialleles, and transposable elements. Imprinted genes show monoallelic expression, whereby only the maternally or paternally inherited allele is expressed [20]. If a gene is "paternally expressed" it means the expressed allele comes from the father and the maternal allele is imprinted (silenced). In regard to growth, paternally expressed genes tend to promote in utero growth, whereas maternally expressed genes restrict growth, and this forms the basis of the "parental conflict theory" [21]. Metastable epialleles (MEs) are genomic loci whose methylation state varies between individuals, but where variation is correlated across tissues originating from all germ layers in a single individual [22]. This indicates that the marks have been laid down in the first few days after conception before cell types start to specialize. MEs therefore provide a useful device to study the influence of the periconceptional environment, including maternal nutrition, on the offspring epigenome [23,24]. Transposable elements (TEs) are small pieces of DNA (usually of viral origin earlier in human history) that are 
mobile and can insert into new chromosomal locations throughout the genome. They are thought to make up more than half of the human genome [25]. TEs arise either through the use of RNA as an intermediate for transposition (DNA is transcribed to RNA, reverse transcribed to DNA, and then inserted by reverse transcriptase to form a retrotransposon), or through the complete DNA sequence being cut and pasted directly (forming DNA transposons, which are less common in the human genome). TEs are potentially functionally disruptive, for example, if transposed into a functional gene or when increasing copy number, and this may be one reason why most are silenced epigenetically [26]. Some TEs are thought to be vulnerable to the influence of nutrition at key time points [27], and their variable methylation patterns have been shown to affect neighboring gene expression, most notably in the Agouti mouse experiments detailed later in this chapter.

\section{EPIGENETICS AND THE DEVELOPMENTAL ORIGINS OF HEALTH AND DISEASE}

The DOHaD hypothesis describes the idea that environmental insults experienced early in life can increase the risk of adverse health outcomes throughout the life course. DOHaD grew out of David Barker's seminal work following a cohort born in Hertfordshire between 1911 and 1930. His early studies found an inverse relationship between birth weight and blood pressure at age 10, with a stronger association at age 36 [28]. His findings soon expanded to identifying associations between low birth weight and adult-onset chronic disease [29], patterns that were also seen in different cohorts such as the Nurses' Health Study I \& II [30] and in Helsinki [31].

Although low birth weight provides a useful proxy for an adverse intrauterine environment, subsequent studies have attempted to pinpoint time points of vulnerability more precisely. Data from famine studies have been particularly useful in this respect. The Dutch Hunger Winter occurred toward the end of World War II, when the Western Netherlands was under German control from November 1944 to May 1945. Nazi blockades cut off food and fuel, and the siege was coupled with a harsh and early winter. Calorie intake varied from 500 to 1,000 kcal per day, depending on the area and time period. An estimated 4.5 million people were affected, 20,000 of whom died [32]. Exposure to famine during pregnancy has been associated with a wide range of offspring phenotypes, from lower birth weight [33] to increased adult blood pressure [34], obesity [35], and risk of schizophrenia [36]. Furthermore the specific timing of famine exposure in utero appears to have different programming effects. For example, exposure in midgestation is associated with a doubling of the prevalence of obesity for men aged 18 to 19, yet exposure in the third trimester is associated with lower obesity [35]. Many of these associations are also found from records spanning the Chinese Great Leap Forward, where famine was particularly severe from 1959 to 1961. For example, famine exposure in utero is associated with a doubled risk of schizophrenia in later life [37], as well as increased hyperglycemia at age 41 to 42, a trend that is exacerbated if an affluent diet was consumed later in life [38].

Although these two famine studies provide rich epidemiological evidence that nutritional exposures in early life are associated with later disease risk, they are unable to determine precise causal factors, for example, if the effects are driven by 
depleted maternal energy, deficient levels of certain micronutrients, or a combination of both. The Pune Maternal Nutrition Study (PMNS) in the Maharashtra State of West-Central India sheds some light on more specific nutritional exposures that may be relevant for DOHaD mechanisms. PMNS is a prospective cohort of 797 women, followed pre- and throughout pregnancy, and their offspring. Higher maternal intake of green leafy vegetables, milk, and fruit, and higher erythrocyte folate concentrations, were associated with larger size babies [39]. Higher maternal folate status at 28 weeks gestation predicted higher offspring adiposity and insulin resistance at the age of 6 , low maternal vitamin $B_{12}$ status was associated with offspring insulin resistance, and a combination of high maternal folate and low $\mathrm{B}_{12}$ produced the strongest associations [40].

Attention is now shifting to an investigation of the mechanisms that may mediate the observations above. In this respect, epigenetic modifications to the genome are emerging as a leading candidate that may, at least partially, explain some of the observations described in the DOHaD literature. For example, Heijmans et al. selected 60 individuals conceived during the Dutch Hunger Winter, and compared them with nonexposed siblings. Five $\mathrm{CpG}$ sites within IGF2 (a maternally imprinted gene controlling fetal growth) were less methylated in individuals exposed to famine at periconception, but there was no difference in methylation for individuals exposed to famine in late gestation [41]. Indeed, the most recent study from the same group confirms that exposure to famine in the first 10 weeks of gestation shows a greater signature in the adult blood methylome (58-59 years) compared to exposure later in gestation [42]. Whilst the exact mechanisms are so far unknown, one concept being debated is the "thrifty epigenome" hypothesis. This proposes that early life insults mold an epigenetic signature to program a phenotype that is "adapted" to the intrauterine environment, which may be problematic if the environment into which the child is born then changes [43]. Under this hypothesis, malnutrition in pregnancy could program "thrifty" epigenotypes, designed to reduce metabolic rate and store energy in an attempt to adapt to a nutritionally poor environment, but may subsequently trigger symptoms of metabolic disease if the environment changes to one of relative nutritional abundance.

\section{MATERNAL NUTRITION EXPOSURES AND THE INFANT EPIGENOME}

There are a variety of factors, both nutrition-related and otherwise, which may impact the infant epigenome in utero through maternal exposure (Figure 22.3). Of these exposures, particular attention has been paid to the role of one-carbon metabolites in the periconceptional period and during embryonic development [44]. In the following section, we give an overview of one-carbon metabolism, and discuss evidence for the influence of one-carbon metabolites on the infant epigenome. We also briefly review other maternal nutrition exposures that have generated research in a human intergenerational setting: maternal BMI, polyunsaturated fatty acids, and vitamin $\mathrm{C}$.

\section{One-Carbon Metabolism}

The one-carbon pool is composed of one-carbon units and the two main carriers that activate, transport, and transfer these units: tetrahydrofolate (THF) and S-adenosyl 


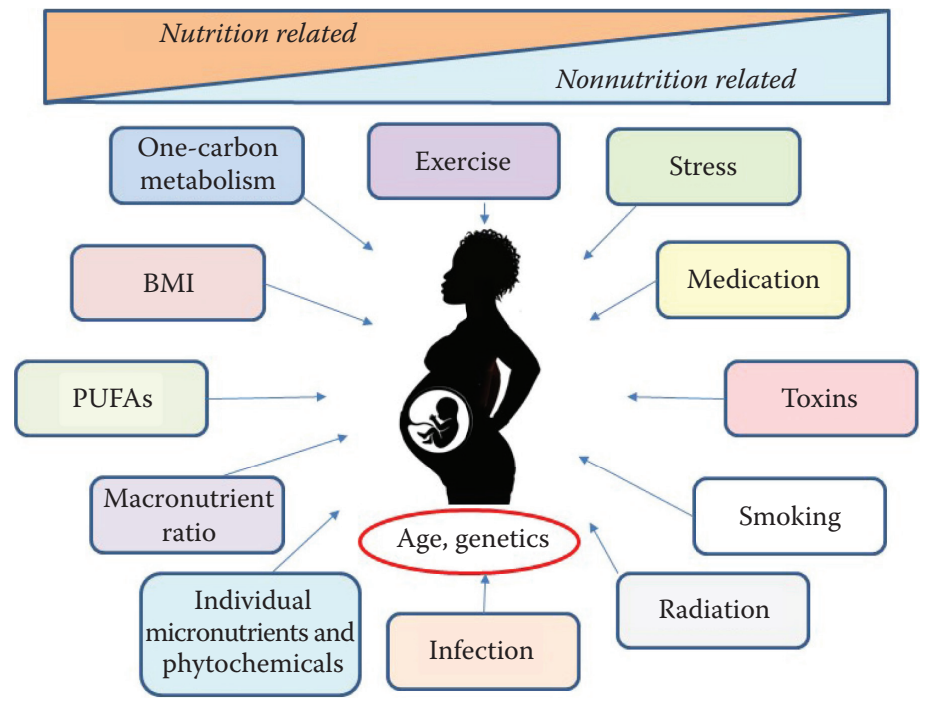

FIGURE 22.3 Potential maternal factors impacting the fetal epigenome.

methionine (SAM). One-carbon units are used as substrates for a whole range of intricate biochemical processes, including cellular biosynthesis, redox status regulation, and genome maintenance through the regulation of nucleotide pools. However, it is their role in transmethylation reactions that is central to the notion of dietepigenome interplay, and this requires an understanding of how folate, methionine, homocysteine, transsulfuration, and transmethylation metabolic pathways interact (Figure 22.4).

S-adenosyl methionine (SAM) methylates a wide variety of acceptors in reactions catalyzed by methyl transferases. Over 200 methylation reactions are required for transcription, translation, protein localization, and signaling purposes [45], but it is the methylation of cytosine bases and amino acids on histone tails that play a role in epigenetics. The donation of SAM's methyl group forms S-adenosyl homocysteine (SAH), which is further hydrolyzed to homocysteine (Hcy), where it is maintained in an equilibrium state that thermodynamically favors SAH over Hcy [46]. A buildup of Hcy results in an increase in $\mathrm{SAH}$, which in turn impedes methylation reactions since SAH competes with SAM for the active site on methyl transferase enzymes [47]. The SAM:SAH ratio is therefore often used as a proxy indicator of methylation potential [48]. In order to maintain favorable methylation conditions, Hcy has to be removed from the system. One way in which this can happen is by accepting a methyl group to form methionine, which can then in turn be condensed with ATP to form SAM and continue the cycle. Hcy can also be removed through its irreversible degradation to cystathionine and cysteine in the transsulfuration pathway requiring vitamin $\mathrm{B}_{6}$.

The methylation of Hcy to methionine uses two distinct pathways. The major pathway is the vitamin $\mathrm{B}_{12}$-dependent reaction involving folate metabolic pathways. A stepwise reduction of dietary folates and folic acid forms tetrahydrofolate (THF). 

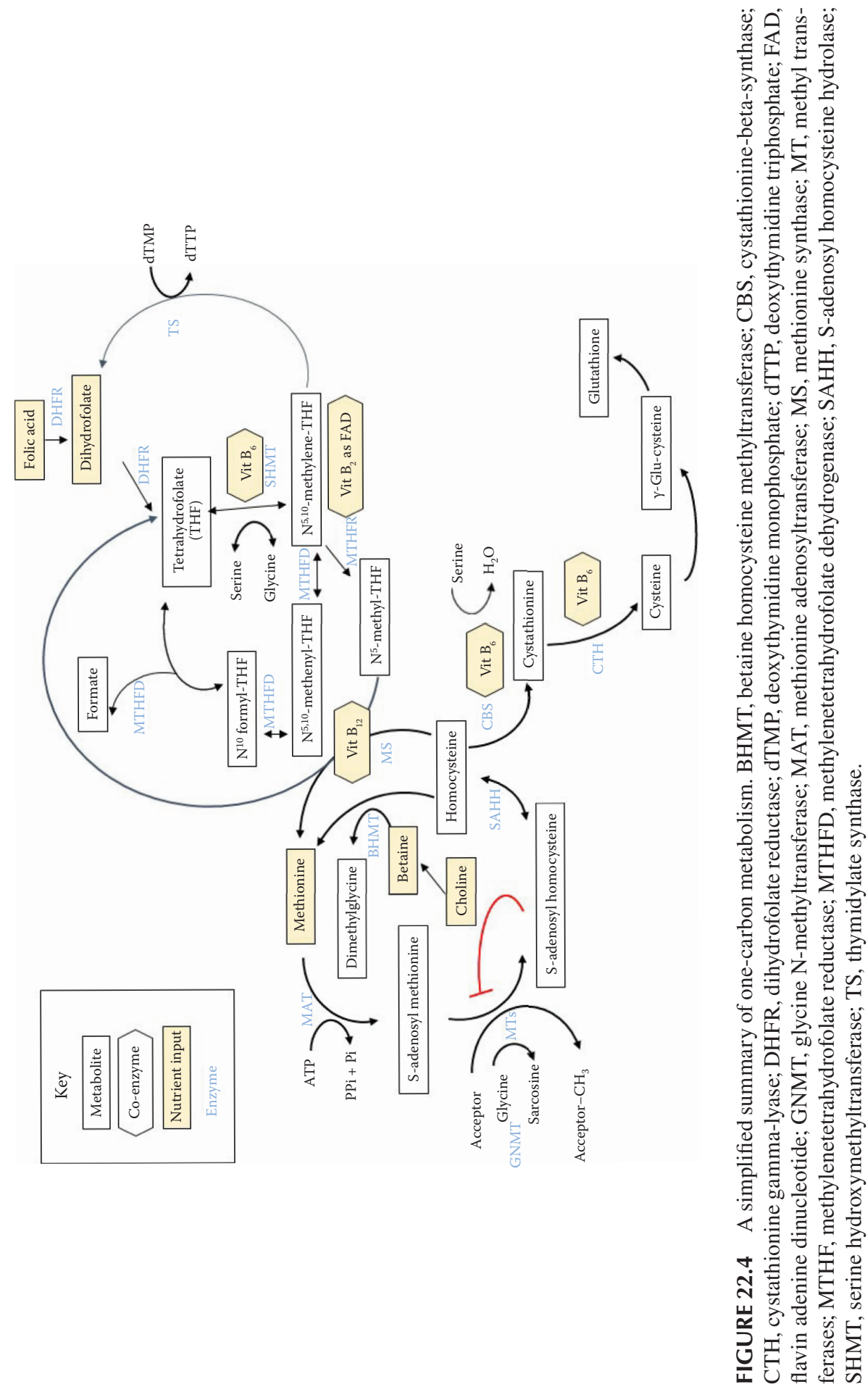
The one-carbon units attach to the $\mathrm{N}^{5}$ or $\mathrm{N}^{10}$ position, or bridge the two. THF can be converted into $\mathrm{N}^{5,10}$-methylene-THF using a methyl group primarily from serine with vitamin $\mathrm{B}_{6}$ as a cofactor. $\mathrm{N}^{5,10}$-methylene-THF is either used in the thymidylate synthase pathway for DNA synthesis and repair, interconverted to $\mathrm{N}^{5,10}$-methenyl-THF, $\mathrm{N}^{10}$-formyl-THF (used in purine synthesis) and formate (an important one-carbon donor) forms, or irreversibly reduced to $\mathrm{N}^{5}$-methyl tetrahydrofolate (methyl-THF) through the action of 5,10-methylene THF reductase (MTHFR) and vitamin $\mathrm{B}_{2}$ as a cofactor. It is methyl-THF that then provides the methyl group for the methylation of Hcy, and in the process regenerates THF. The alternative pathway for the methylation of Hcy, predominantly used in the liver and kidneys, uses the methyl group from betaine, a product formed through the oxidation of choline. This description and Figure 22.4 give a very simplified overview of the complex and interlinking metabolic pathways involved, some of which occur with different enzymes in different cellular compartments [49]. However, this brief overview helps explain why deficiencies in the key methyl donors of choline, betaine, or folate, alongside deficiencies in vitamins $\mathrm{B}_{2}, \mathrm{~B}_{6}$, and $\mathrm{B}_{12}$, which play essential coenzyme roles, may disrupt the metabolic pathways that are responsible for DNA methylation. Genetic variants in proteins coding for enzymes used in key one-carbon metabolic processes can also affect activity at critical nodes and alter the flow of metabolites [50].

\section{Studies Investigating One-Carbon Metabolites and Epigenetics}

Perhaps the most famous animal experiments demonstrating how epigenetic changes driven by maternal diet in pregnancy can dramatically alter phenotype in the offspring come from the agouti mouse. In one experiment, pregnant dams were fed a diet that varied in methyl donor content (folic acid, choline, betaine, and vitamin $\mathrm{B}_{12}$ ). Their isogenic pups showed variable methylation at an intracisternal A particle (IAP), a retrotransposon upstream of the agouti gene that is a metastable epiallele. The degree of methylation at this locus altered expression of the agouti gene, resulting in permanent phenotypic differences. The most obvious change was in fur color, but differences were also found in appetite, adiposity, and glucose tolerance, factors highly relevant to life-long chronic disease risk [19,27]. A similar experiment in a different strain of kinky-tailed mice showed that methyl donor content of the maternal diet also altered methylation at an IAP on the Axin gene, producing pups with varying levels of tail kink [51].

Human studies exploring associations between individual one-carbon metabolites and epigenetic effects are few and far between, especially those involving an intergenerational study design. Homocysteine and vitamin $\mathrm{B}_{6}$ are two examples that are described in human cross-sectional studies assessing immediate effects on health at one time point within a single generation. Hyperhomocysteinemia has been associated with hypomethylation in several studies [52] supporting the hypothesis that epigenetic mechanisms may play a partial role in observed associations between hyperhomocysteinemia and decreased cardiovascular health via inflammation, free radical formation, and atherogenesis [53]. Low vitamin $\mathrm{B}_{6}$ levels may decrease $\mathrm{N}^{5,10}$-methenyl-THF through reduced serine hydroxymethyltransferase (SHMT) activity (Figure 22.4), stressing the thymidylate synthase pathway for DNA repair and incorporating more 
uracil into DNA. This reduces genome stability through increased chromosome breaks, which, in turn, have been associated with increased levels of global DNA hypomethylation alongside a putative increased risk of tumor development [54].

Of the human studies using an intergenerational design, folate, vitamin $\mathrm{B}_{12}$, and choline have been most frequently investigated. Due to its role in preventing neural tube defects and involvement in some cancer therapies, folic acid has been extensively reviewed [55]. Increased periconceptional folic acid consumption has been associated with increased methylation in infants at a differentially methylated region (DMR) of the IGF2 gene, although these associations have not been established consistently [56]. Additional studies have found that maternal folate levels in late pregnancy influence offspring methylation patterns in the imprinting control region of IGF2 [57] and also in the imprinting regulator ZFP57 [58]. Despite the timing of maternal folate measurements, these findings support the importance of nutritional status around conception, since many DMRs appear to have their methylation pattern established prior to gastrulation [59]. There are fewer studies assessing the effect of maternal vitamin $B_{12}$ status on the infant epigenome. However, preliminary evidence suggests that maternal vitamin $B_{12}$ levels at first antenatal visit are inversely correlated with infant cord blood global methylation levels [60]. For choline, one intervention study investigated the effect of supplementing mothers in the third trimester with daily choline of $480 \mathrm{mg}$ versus $930 \mathrm{mg}$ [61]. Higher maternal choline intake was associated with increased methylation of $\mathrm{CRH}$ and $\mathrm{NR} 3 \mathrm{Cl}$ promoter regions within fetal placental tissue.

The complex interlinking metabolic pathways involved in one-carbon metabolism may be more appropriately investigated by exploring the joint effects of relevant methyl donors and coenzymes. Researchers in The Gambia have been exploring human diet-epigenome interactions by exploiting a natural experimental design, in which fluctuations in energy balance and maternal nutritional exposures display a distinct bimodal seasonal pattern. The Gambia experiences a rainy ("hungry") season from July to September (Figure 22.5a), a planting season with increased energy expenditure, depleted food stores, and peaks of malarial and diarrheal diseases. The dry ("harvest") season occurs from February to April (Figure 22.5b), when

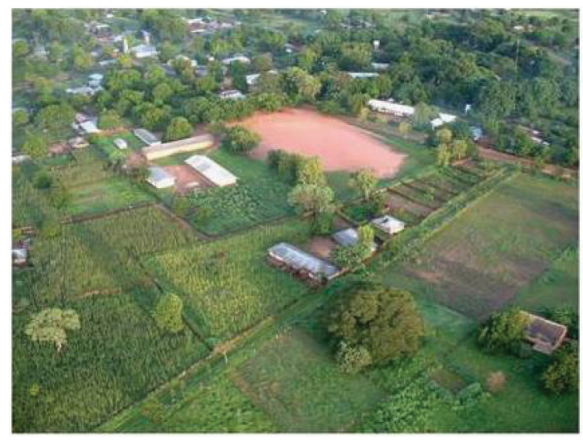

(a)

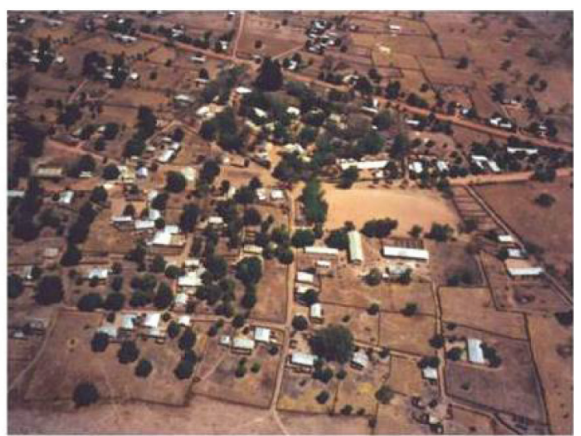

(b)

FIGURE 22.5 The Gambian (a) rainy/"hungry" and (b) dry/harvest seasons in Keneba, West Kiang. (Photo by Andrew M. Prentice.) 
harvesting takes place, leading to improved food security. Despite overall household food stores being more replete in the dry season, the rainy season offers the increased availability of certain micronutrients made available from rain-fed green leafy vegetables. It has been known for several decades that Gambian children born during the rainy season are up to 10 times more likely to die prematurely in young adulthood than those born during the dry season [62]. However, the processes underlying these findings have yet to be fully delineated, and nutrition-related epigenetic regulation in early embryonic development is emerging as one plausible contributory mechanism. A recent study compared women conceiving in the peak of the dry and the peak of the rainy season [24]. Maternal periconceptional plasma concentrations of folate, vitamin $\mathrm{B}_{2}$, methionine, betaine, and the SAM:SAH and betaine:dimethyl glycine ratios were higher in the rainy season, and concentrations of vitamin $B_{12}$, homocysteine, and SAH were lower. This suggested that the maternal metabolome during the rainy season contained higher concentrations of methyl donors and exhibited a higher methylation potential than the dry season metabolome. Indeed, offspring of these rainy season conceptions had higher levels of $\mathrm{CpG}$ methylation at six MEs in peripheral blood monocytes compared to the offspring of those conceived in the dry season, with similar patterns in hair follicle DNA [24]. A subsequent study details the genome-wide search for MEs susceptible to the periconceptional environment using two independent approaches [63]. Both found VTRNA2-1 as their top hit, an imprinted gene that was also found to exhibit increased hypomethylation among Gambian offspring conceived in the dry season compared to the rainy season. Taken together, the results support the hypothesis that maternal nutrition during the periconceptional period can impact the infant epigenome in humans and has triggered further research into the possible phenotypic consequences.

\section{Polyunsaturated Fatty Acids}

Several studies indicate that increased consumption of $\omega-3$ PUFAs is associated with reduced homocysteine levels [64]. In the context of early life nutrition, maternal fatty acid intake influences infant fatty acid composition via placental transfer and breast milk, with maternal PUFA intake potentially affecting infant appetite control, neuroendocrine function, and metabolic programming [65]. Investigating whether one-carbon pathways and epigenetic mechanisms are involved is of increasing interest. In rodent models, providing a maternal diet with excess folic acid and restricted vitamin $B_{12}$ (designed to reflect the nutritional situation in much of rural India) is associated with increased maternal oxidative stress, lower level of offspring placental and brain docosahexaenoic acid, and decreased placental global methylation [66]. However, maternal supplementation with PUFAs seems to partially ameliorate the disrupted one-carbon metabolism associated with this diet [67]. One potential mechanism is through PUFAs upregulating enzymes responsible for the methylation of homocysteine to methionine [68], which in theory could increase the SAM:SAH ratio and help overcome the impact of reduced MTHFR activity brought about by the restricted vitamin $\mathrm{B}_{12}$. An additional recent hypothesis describes how a decreased maternal PUFA intake may increase the availability of methyl groups for DNA methylation since there would be reduced PUFAs for phosphatidylcholine synthesis and therefore less demand for methyl groups for this 
pathway. The disruption to the one-carbon pathways brought about by low PUFA levels is thought to alter epigenetic programming of placental genes, increasing the risk of aberrant methylation in offspring and adverse pregnancy outcome [69]. Human evidence at the intergenerational level, however, is currently limited to a study by Lee et al. [70]. Here, mothers were given either $400 \mathrm{mg}$ of $\omega$-3 PUFA $(n=131)$ or placebo $(n=130)$ daily from approximately 18 weeks gestation to birth. Among mothers supplemented with PUFAs, there was increased global methylation (measured at LINE-1 repetitive elements) in offspring of mothers who smoked ( $n=26$ case, 26 control) [71], and increased offspring $I G F 2$ promoter 3 methylation in preterm infants ( $n=16$ case, 20 control) [70].

\section{Body Mass IndeX}

Maternal body mass index (BMI) shows some association with infant epigenetic patterns [72]. However, it can be difficult to define exactly what BMI represents as an exposure, since it does not always correlate well with body composition [73] and is a proxy for a wide range of potential metabolic disorders [74]. In an intergenerational setting, both maternal underweight and overweight is associated with epigenetic patterns in infant cord blood [72]. The mechanism by which BMI influences epigenetics is so far unknown. Although SAM has been shown to be independently, positively associated with fat mass in older adults, alongside a higher rate of converting methionine into SAM in obese individuals, the absence of a rise in the SAM:SAH ratio means conclusions are hard to draw [75]. Paternal BMI may also be a relevant exposure to consider, since neonates born to obese fathers in a U.S. study showed hypomethylation at some loci within imprinted genes (MEST, PEG3, NNAT), independent of maternal obesity [76].

\section{Vitamin C and Demethylation}

As outlined earlier, the periconceptional period is a time of widespread remodeling of the offspring epigenome, including rapid erasure (demethylation) of parental epigenetic marks and subsequent remethylation (Figure 22.2). In demethylation, 5-methylcytosine $(5 \mathrm{mC})$ can be sequentially oxidized to different states by teneleven translocation (TET) dioxygenases that use vitamin C (ascorbate) as a cofactor [77]. In vitro studies, for example, indicate that adding vitamin $\mathrm{C}$ to mouse or human embryonic stem cells increases activity of TET enzymes, with active demethylation seen in the germline and associated gene expression changes [78]. Vitamin $\mathrm{C}$ is therefore an important factor to consider in epigenetic processes during early embryogenesis, although in vivo studies will be required to explore whether vitamin $\mathrm{C}$ deficiency could play a role in aberrant demethylation.

In this section we have identified some of the key ways that maternal nutrition might influence the offspring epigenome. Other exposures, both nutrition-related and otherwise, may also be involved. These include maternal stress [79], toxin exposure [80], maternal hyperglycemia [81], the microbiome [82], dietary polyphenols [83], vitamin D [84], vitamin A [85], and infection [86]. As evidence builds, it will be necessary to explore how these multiple exposures work together to influence the 
infant epigenome and to more clearly define the potential consequences for human health. It is to the latter point that we now turn.

\section{PHENOTYPES ASSOCIATED WITH THE IMPACT OF MATERNAL EXPOSURES ON THE INFANT EPIGENOME}

Most population-based epigenetic studies to date have been limited to exploring either associations between maternal exposures and infant epigenetic patterns, or between infant epigenetic patterns and later phenotypes. Evidence linking maternal exposure to infant phenotypes with epigenetics as a mediating mechanism is scarce.

The first category of associations, linking maternal exposures to infant epigenetic patterns, was reviewed earlier. A number of studies linking infant epigenetic patterns with later phenotypes have focused on imprinted genes as candidate loci, due to their role in fetal growth [17] and a range of diseases [3,20]. It has been suggested that imprinted genes may be more vulnerable to the effects of epigenetic dysregulation, since epigenetic mechanisms underpin their monoallelic expression. On chromosome region 11p15.5 there are two imprinting control regions (ICRs) of interest: the H19/IGF2 domain (ICR1) and the KCNQ1/CDKN1C domain (ICR2). Hypomethylation of ICR1 and hypermethylation of ICR2 are associated with Russell-Silver syndrome (RSS; an undergrowth disorder), whereas hypermethylation of ICR1 and hypomethylation of ICR2 are associated with Beckwith-Wiedemann syndrome (BWS; an overgrowth disorder) [3]. In addition to the aberrant methylation found at ICR1 and ICR2, some studies suggest patients with RSS and BWS show methylation defects at multiple gene loci [87]. Evidence for associations between growth-related phenotypes (other than RSS and BWS) and methylation patterns at several imprinted gene loci is slowly accumulating. Other phenotypes investigated to date include intrauterine growth restriction [88], small for gestational age [89], birth weight [90] and later adiposity [91].

Several studies describe associations between maternal one-carbon metabolites and infant growth-related outcomes, for example, vitamin $B_{12}$ [92], folate [93], and homocysteine [94]. What then is the evidence that these associations are mediated through epigenetic mechanisms? Preliminary evidence from the Dutch Hunger Winter, as described earlier, suggests that exposure to famine in pregnancy, particularly around conception, is associated with differential methylation in genes linked to growth, and development [41], and that famine exposure is also related to a wide range of offspring cognitive health and cardiometabolic risk factors six decades later [95]. It is, however, difficult to establish the direction of causality, since disease states can also influence the epigenome [96]. This issue of reverse causality is particularly pertinent to studies using a retrospective cohort design. Stronger evidence comes from prospective cohorts, such as the Newborn Epigenetics Study (NEST) in the United States. Hoyo et al. describe a positive association between maternal folate levels in the first trimester and birth weight [90]. Increased maternal folate was also associated with increased methylation at MEG3, PLAGL1, and PEG3 in infant cord blood, and decreased methylation at $I G F 2$. Five differentially methylated sites were associated with birth weight, and it was speculated that the association seen between maternal 
folate and birth weight could be mediated by differential methylation at MEG3, H19, and PLAGL1. In a more recent study from the same cohort, McCullough et al. found maternal plasma concentrations of homocysteine in the first trimester were inversely associated with birth weight, particularly in males [97]. Children born to mothers with the highest quartile of plasma $\mathrm{B}_{12}$ showed lower weight gain between birth and 3 years. However, only maternal vitamin $B_{6}$ was positively associated with cord methylation at a DMR from MEG3. A further example comes from Godfrey et al., who found that higher methylation of RXRA and eNOS in umbilical cord tissue was associated with offspring adiposity at age 9 , and that higher $R X R A$ methylation was also associated with lower maternal carbohydrate intake in early pregnancy [91].

Given the scarcity of existing data and the many potential confounders in cohort studies, there is not yet any clear consensus on the extent to which epigenetics is involved in the etiology of suboptimal growth, particularly in the more widely investigated phenotypes of IUGR and SGA [98]. Similarly, although prepregnancy folic acid supplementation is known to prevent neural tube defects, the extent to which this protection is epigenetically mediated is not yet clear [56]. In the next few years, a number of prospective cohort studies should shed further light on the links between early life exposures and phenotypes, mediated by epigenetic mechanisms. In the meantime, we can use the literature from the range of studies described earlier to speculate that periconceptional nutritional insults may influence epigenetic mechanisms in the infant, causing disruption at multiple loci and potentially leading to a broad spectrum of phenotypic consequences in later life.

\section{NEXT STEPS}

The field of epigenetic epidemiology is in its infancy. Future challenges include the need to consider a much larger number of candidate genetic regions (a challenge that will be met as rapid improvements in technology facilitate epigenome-wide association studies with ever increasing genomic resolution); the need to focus on tissue types most appropriate to the phenotype of interest; and the need to integrate data on genetic variation, gene expression, and other epigenetic mechanisms such as histone modifications and miRNAs. Investigations will also need to assess the extent to which postnatal exposures may interact with periconceptional effects. Finally, although we focus on the maternal periconceptional environment in this chapter, there is growing interest in the potential for paternal exposures to affect the infant epigenome transgenerationally via sperm methylation profiles [99]. Despite limited human evidence to date, this is an area of great potential public heath relevance.

Once disrupted epigenetic patterns can be better mapped onto phenotypes, the significance for public health impact becomes more tangible. Can we design and test nutritional interventions to "correct" a suboptimal maternal metabolome, hence reducing patterns of aberrant methylation in the infant epigenome and reducing the burden of ill health? Despite the current complexities of disentangling the web of multiple exposures influencing the infant epigenome, as technology advances, collaborations grow, and knowledge is gained, the field of nutritional epigenetics has the potential to impact the health of children not only within the first 1,000 days but also across multiple generations. 


\section{REFERENCES}

1. Egger G, Liang G, Aparicio A et al. Epigenetics in human disease and prospects for epigenetic therapy. Nature 2004; 429:457-63.

2. Esteller M. Epigenetics in cancer. N Engl J Med 2008; 358:1148-59.

3. Piedrahita JA. The role of imprinted genes in fetal growth abnormalities. Birth Defects Res A Clin Mol Teratol 2011; 91:682-92.

4. Jaenisch R, Bird A. Epigenetic regulation of gene expression: How the genome integrates intrinsic and environmental signals. Nat Genet 2003; 33(Suppl):245-54.

5. Wang Y, Leung FCC. An evaluation of new criteria for $\mathrm{CpG}$ islands in the human genome as gene markers. Bioinformatics 2004; 20:1170-7.

6. Illingworth RS, Bird AP. CpG islands: “A rough guide.” FEBS Lett 2009; 583:1713-20.

7. Hill PWS, Amouroux R, Hajkova P. DNA demethylation, Tet proteins and 5-hydroxymethylcytosine in epigenetic reprogramming: An emerging complex story. Genomics 2014; 104:324-33.

8. Messerschmidt DM, Knowles BB, Solter D. DNA methylation dynamics during epigenetic reprogramming in the germline and preimplantation embryos. Genes Dev 2014; 28:812-28.

9. Kouzarides T. Chromatin modifications and their function. Cell 2007; 128:693-705.

10. Klose RJ, Bird AP. Genomic DNA methylation: The mark and its mediators. Trends Biochem Sci 2006; 31:89-97.

11. Richards EJ, Elgin SC. Epigenetic codes for heterochromatin formation and silencing. Cell 2002; 108:489-500.

12. Mattick JS, Makunin I V. Non-coding RNA. Hum Mol Genet 2006; 15(Spec No 1):R17-29.

13. Guil S, Esteller M. DNA methylomes, histone codes and miRNAs: Tying it all together. Int J Biochem Cell Biol 2009; 41:87-95.

14. Sato F, Tsuchiya S, Meltzer SJ et al. MicroRNAs and epigenetics. FEBS J 2011; 278:1598-609.

15. Langley-Evans SC. Nutrition in early life and the programming of adult disease: A review. J Hum Nutr Diet 2015; 28:1-14.

16. Seisenberger S, Peat JR, Hore TA et al. Reprogramming DNA methylation in the mammalian life cycle: Building and breaking epigenetic barriers. Philos Trans $R$ Soc Lond B Biol Sci 2013; 368:20110330.

17. Reik W, Walter J. Genomic imprinting: Parental influence on the genome. Nat Rev Genet 2001; 2:21-32.

18. Smallwood SA, Kelsey G. De novo DNA methylation: A germ cell perspective. Trends Genet 2012; 28:33-42.

19. Waterland RA, Michels KB. Epigenetic epidemiology of the developmental origins hypothesis. Annu Rev Nutr 2007; 27:363-88.

20. Ishida M, Moore GE. The role of imprinted genes in humans. Mol Aspects Med 2013; 34:826-40.

21. Moore GE, Ishida M, Demetriou $\mathrm{C}$ et al. The role and interaction of imprinted genes in human fetal growth. Philos Trans R Soc B Biol Sci 2015; 370:20140074.

22. Rakyan VK, Blewitt ME, Druker R et al. Metastable epialleles in mammals. Trends Genet 2002; 18:348-51.

23. Waterland RA, Kellermayer R, Laritsky E et al. Season of conception in rural Gambia affects DNA methylation at putative human metastable epialleles. PLoS Genet 2010; 6:e1001252.

24. Dominguez-Salas P, Moore SE, Baker MS et al. Maternal nutrition at conception modulates DNA methylation of human metastable epialleles. Nat Commun 2014; 5:3746. 
25. Lander ES, Linton LM, Birren B et al. Initial sequencing and analysis of the human genome. Nature 2001; 409:860-921.

26. Slotkin RK, Martienssen R. Transposable elements and the epigenetic regulation of the genome. Nat Rev Genet 2007; 8:272-85.

27. Waterland RA, Jirtle RL. Transposable elements: Targets for early nutritional effects on epigenetic gene regulation. Mol Cell Biol 2003; 23:5293-300.

28. Barker DJ, Osmond C, Golding J et al. Growth in utero, blood pressure in childhood and adult life, and mortality from cardiovascular disease. BMJ 1989; 298:564-7.

29. Barker DJ, Gluckman PD, Godfrey KM et al. Fetal nutrition and cardiovascular disease in adult life. Lancet (London, England) 1993; 341:938-41.

30. Curhan GC, Chertow GM, Willett WC et al. Birth weight and adult hypertension and obesity in women. Circulation 1996; 94:1310-5.

31. Eriksson JG, Forsen T, Tuomilehto J et al. Catch-up growth in childhood and death from coronary heart disease: Longitudinal study. BMJ 1999; 318:427-31.

32. Lumey LH, Stein AD, Kahn HS et al. Cohort profile: The Dutch Hunger Winter families study. Int J Epidemiol 2007; 36:1196-1204.

33. Smith CA. The effect of wartime starvation in Holland upon pregnancy and its product. Am J Obstet Gynecol 1947; 53:599-608.

34. Roseboom TJ, van der Meulen JH, van Montfrans GA et al. Maternal nutrition during gestation and blood pressure in later life. J Hypertens 2001; 19:29-34.

35. Ravelli GP, Stein ZA, Susser MW. Obesity in young men after famine exposure in utero and early infancy. N Engl J Med 1976; 295:349-53.

36. Susser E, Neugebauer R, Hoek HW et al. Schizophrenia after prenatal famine. Further evidence. Arch Gen Psychiatry 1996; 53:25-31.

37. St Clair D, Xu M, Wang P et al. Rates of adult schizophrenia following prenatal exposure to the Chinese famine of 1959-1961. JAMA 2005; 294:557-62.

38. Li Y, He Y, Qi L et al. Exposure to the Chinese famine in early life and the risk of hyperglycemia and type 2 diabetes in adulthood. Diabetes 2010; 59:2400-6.

39. Rao S, Yajnik CS, Kanade A et al. Intake of micronutrient-rich foods in rural Indian mothers is associated with the size of their babies at birth: Pune Maternal Nutrition Study. J Nutr 2001; 131:1217-24.

40. Yajnik CS, Deshpande SS, Jackson AA et al. Vitamin $B_{12}$ and folate concentrations during pregnancy and insulin resistance in the offspring: The Pune Maternal Nutrition Study. Diabetologia 2008; 51:29-38.

41. Heijmans BT, Tobi EW, Stein AD et al. Persistent epigenetic differences associated with prenatal exposure to famine in humans. Proc Natl Acad Sci USA 2008; 105:17046-9.

42. Tobi EW, Slieker RC, Stein AD et al. Early gestation as the critical time-window for changes in the prenatal environment to affect the adult human blood methylome. Int $J$ Epidemiol 2015; 44:1211-23.

43. Stöger R. The thrifty epigenotype: An acquired and heritable predisposition for obesity and diabetes? BioEssays 2008; 30:156-66.

44. Steegers-Theunissen RPM, Twigt J, Pestinger V et al. The periconceptional period, reproduction and long-term health of offspring: The importance of one-carbon metabolism. Hum Reprod Update 2013; 19:640-55.

45. Lu SC, Mato JM. S-adenosylmethionine in liver health, injury, and cancer. Physiol Rev 2012; 92:1515-42.

46. Pajares MA, Pérez-Sala D. Betaine homocysteine S-methyltransferase: Just a regulator of homocysteine metabolism? Cell Mol Life Sci 2006; 63:2792-803.

47. Scotti M, Stella L, Shearer EJ et al. Modeling cellular compartmentation in one-carbon metabolism. Wiley Interdiscip Rev Syst Biol Med 2013; 5:343-65. 
48. Mason JB. Biomarkers of nutrient exposure and status in one-carbon (methyl) metabolism. J Nutr 2003; 133:941S-947.

49 Stover PJ, Field MS. Trafficking of intracellular folates. Adv Nutr 2011; 2:325-31.

50. Fredriksen A, Meyer K, Ueland PM et al. Large-scale population-based metabolic phenotyping of thirteen genetic polymorphisms related to one-carbon metabolism. Hum Mutat 2007; 28:856-65.

51. Waterland RA, Dolinoy DC, Lin J-R et al. Maternal methyl supplements increase offspring DNA methylation at Axin Fused. Genesis 2006; 44:401-6.

52. Zhou S, Zhang Z, Xu G. Notable epigenetic role of hyperhomocysteinemia in atherogenesis. Lipids Health Dis 2014; 13:134.

53. Beard RS, Bearden SE. Vascular complications of cystathionine $\beta$-synthase deficiency: Future directions for homocysteine-to-hydrogen sulfide research. Am J Physiol Heart Circ Physiol 2011; 300:H13-26.

54. Huang JY, Butler LM, Wang R et al. Dietary intake of one-carbon metabolism-related nutrients and pancreatic cancer risk: The Singapore Chinese Health Study. Cancer Epidemiol Biomarkers Prev 2016; 25:417-24.

55. Crider KS, Yang TP, Berry RJ et al. Folate and DNA methylation: A review of molecular mechanisms and the evidence for folate's role. Adv Nutr 2012; 3:21-38.

56. Gonseth S, Roy R, Houseman EA et al. Periconceptional folate consumption is associated with neonatal DNA methylation modifications in neural crest regulatory and cancer development genes. Epigenetics 2015; 10:1166-76.

57. Ba Y, Yu H, Liu F et al. Relationship of folate, vitamin $B_{12}$ and methylation of insulinlike growth factor-II in maternal and cord blood. Eur J Clin Nutr 2011; 65:480-5.

58. Amarasekera M, Martino D, Ashley S et al. Genome-wide DNA methylation profiling identifies a folate-sensitive region of differential methylation upstream of ZFP57imprinting regulator in humans. FASEB J 2014; 28:4068-76.

59. Woodfine K, Huddleston JE, Murrell A. Quantitative analysis of DNA methylation at all human imprinted regions reveals preservation of epigenetic stability in adult somatic tissue. Epigenetics Chromatin 2011; 4:1.

60. McKay JA, Groom A, Potter C et al. Genetic and non-genetic influences during pregnancy on infant global and site specific DNA methylation: Role for folate gene variants and vitamin $\mathrm{B}_{12}$. PLoS One 2012; 7:e33290.

61. Jiang X, Yan J, West AA et al. Maternal choline intake alters the epigenetic state of fetal cortisol-regulating genes in humans. FASEB J 2012; 26:3563-74.

62. Moore SE, Cole TJ, Poskitt EME et al. Season of birth predicts mortality in rural Gambia. Nature 1997; 388:434.

63. Silver MJ, Kessler NJ, Hennig BJ et al. Independent genomewide screens identify the tumor suppressor VTRNA2-1 as a human epiallele responsive to periconceptional environment. Genome Biol 2015; 16:118.

64. Huang $\mathrm{T}$, Zheng $\mathrm{J}$, Chen $\mathrm{Y}$ et al. High consumption of $\Omega-3$ polyunsaturated fatty acids decrease plasma homocysteine: A meta-analysis of randomized, placebo-controlled trials. Nutrition 2011; 27:863-7.

65. Kabaran S, Besler HT. Do fatty acids affect fetal programming? J Heal Popul Nutr 2015; 33:14.

66. Roy S, Kale A, Dangat K et al. Maternal micronutrients (folic acid and vitamin B(12)) and omega 3 fatty acids: Implications for neurodevelopmental risk in the rat offspring. Brain Dev 2012; 34:64-71.

67. Khot V, Kale A, Joshi A et al. Expression of genes encoding enzymes involved in the one carbon cycle in rat placenta is determined by maternal micronutrients (folic acid, vitamin $\mathrm{B}_{12}$ ) and omega-3 fatty acids. Biomed Res Int 2014; 2014:613078. 
68. Huang T, Hu X, Khan N et al. Effect of polyunsaturated fatty acids on homocysteine metabolism through regulating the gene expressions involved in methionine metabolism. Sci World J 2013; 2013:1-8.

69. Khot V, Chavan-Gautam P, Joshi S. Proposing interactions between maternal phospholipids and the one carbon cycle: A novel mechanism influencing the risk for cardiovascular diseases in the offspring in later life. Life Sci 2015; 129:16-21.

70. Lee H-S, Barraza-Villarreal A, Biessy C et al. Dietary supplementation with polyunsaturated fatty acid during pregnancy modulates DNA methylation at IGF2/H19 imprinted genes and growth of infants. Physiol Genomics 2014; 46:851-7.

71. Lee H-S, Barraza-Villarreal A, Hernandez-Vargas H et al. Modulation of DNA methylation states and infant immune system by dietary supplementation with $\omega$-3 PUFA during pregnancy in an intervention study. Am J Clin Nutr 2013; 98:480-7.

72. Sharp GC, Lawlor DA, Richmond RC et al. Maternal pre-pregnancy BMI and gestational weight gain, offspring DNA methylation and later offspring adiposity: Findings from the Avon Longitudinal Study of Parents and Children. Int J Epidemiol 2015; 44:1288-304.

73. Wells JCK, Fewtrell MS. Measuring body composition. Arch Dis Child 2006; 91:612-7.

74. Müller MJ, Lagerpusch M, Enderle J et al. Beyond the body mass index: Tracking body composition in the pathogenesis of obesity and the metabolic syndrome. Obes Rev 2012; 13(Suppl 2):6-13.

75. Elshorbagy AK, Nijpels G, Valdivia-Garcia M et al. S-adenosylmethionine is associated with fat mass and truncal adiposity in older adults. J Nutr 2013; 143:1982-8.

76. Soubry A, Murphy SK, Wang F et al. Newborns of obese parents have altered DNA methylation patterns at imprinted genes. Int J Obes 2015; 39:650-7.

77. Young JI, Züchner S, Wang G. Regulation of the epigenome by vitamin C. Annu Rev Nutr 2015; 35:545-64.

78. Blaschke K, Ebata KT, Karimi MM et al. Vitamin C induces Tet-dependent DNA demethylation and a blastocyst-like state in ES cells. Nature 2013; 500:222-6.

79. Babenko O, Kovalchuk I, Metz GAS. Stress-induced perinatal and transgenerational epigenetic programming of brain development and mental health. Neurosci Biobehav Rev 2014; 48:70-91.

80. Anway MD, Skinner MK. Epigenetic transgenerational actions of endocrine disruptors. Endocrinology 2006; 147:S43-49.

81. El Hajj N, Schneider E, Lehnen H et al. Epigenetics and life-long consequences of an adverse nutritional and diabetic intrauterine environment. Reproduction 2014; 148:R111-20.

82. Davie JR. Inhibition of histone deacetylase activity by butyrate. J Nutr 2003; 133:2485S-93S.

83. Fang M, Chen D, Yang CS. Dietary polyphenols may affect DNA methylation. $J$ Nutr 2007; 137:223S-8S.

84. Pereira F, Barbáchano A, Singh PK et al. Vitamin D has wide regulatory effects on histone demethylase genes. Cell Cycle 2012; 11:1081-9.

85. Feng Y, Zhao L-Z, Hong L et al. Alteration in methylation pattern of GATA-4 promoter region in vitamin A-deficient offspring's heart. J Nutr Biochem 2013; 24:1373-80.

86. Claycombe KJ, Brissette CA, Ghribi O. Epigenetics of inflammation, maternal infection, and nutrition. J Nutr 2015; 145:1109S-15S.

87. Azzi S, Rossignol S, Steunou V et al. Multilocus methylation analysis in a large cohort of 11p15-related foetal growth disorders (Russell Silver and Beckwith Wiedemann syndromes) reveals simultaneous loss of methylation at paternal and maternal imprinted loci. Hum Mol Genet 2009; 18:4724-33.

88. Einstein F, Thompson RF, Bhagat TD et al. Cytosine methylation dysregulation in neonates following intrauterine growth restriction. PLoS One 2010; 5:e8887. 
89. Bouwland-Both MI, van Mil NH, Stolk L et al. DNA methylation of IGF2DMR and H19 is associated with fetal and infant growth: The generation R study. PLoS One 2013; 8:e81731.

90. Hoyo C, Daltveit AK, Iversen E et al. Erythrocyte folate concentrations, CpG methylation at genomically imprinted domains, and birth weight in a multiethnic newborn cohort. Epigenetics 2014; 9:1120-30.

91. Godfrey KM, Sheppard A, Gluckman PD et al. Epigenetic gene promoter methylation at birth is associated with child's later adiposity. Diabetes 2011; 60:1528-34.

92. Rush EC, Katre P, Yajnik CS. Vitamin $\mathrm{B}_{12}$ : One carbon metabolism, fetal growth and programming for chronic disease. Eur J Clin Nutr 2014; 68:2-7.

93. van Uitert EM, Steegers-Theunissen RPM. Influence of maternal folate status on human fetal growth parameters. Mol Nutr Food Res 2013; 57:582-95.

94. Hogeveen M, Blom HJ, den Heijer M. Maternal homocysteine and small-for-gestationalage offspring: Systematic review and meta-analysis. Am J Clin Nutr 2012; 95:130-6.

95. de Rooij SR, Painter RC, Roseboom TJ et al. Glucose tolerance at age 58 and the decline of glucose tolerance in comparison with age 50 in people prenatally exposed to the Dutch famine. Diabetologia 2006; 49:637-43.

96. Relton CL, Davey Smith G. Two-step epigenetic Mendelian randomization: A strategy for establishing the causal role of epigenetic processes in pathways to disease. Int J Epidemiol 2012; 41:161-76.

97. McCullough LE, Miller EE, Mendez MA et al. Maternal B vitamins: Effects on offspring weight and DNA methylation at genomically imprinted domains. Clin Epigenetics 2016; 8:8.

98. Toure DM, Baccaglini L, Opoku ST et al. Epigenetic dysregulation of Insulin-like growth factor (IGF)-related genes and adverse pregnancy outcomes: A systematic review. J Matern Fetal Neonatal Med 2016; 18:1-11.

99. Soubry A. Epigenetic inheritance and evolution: A paternal perspective on dietary influences. Prog Biophys Mol Biol 2015; 118:79-85. 